
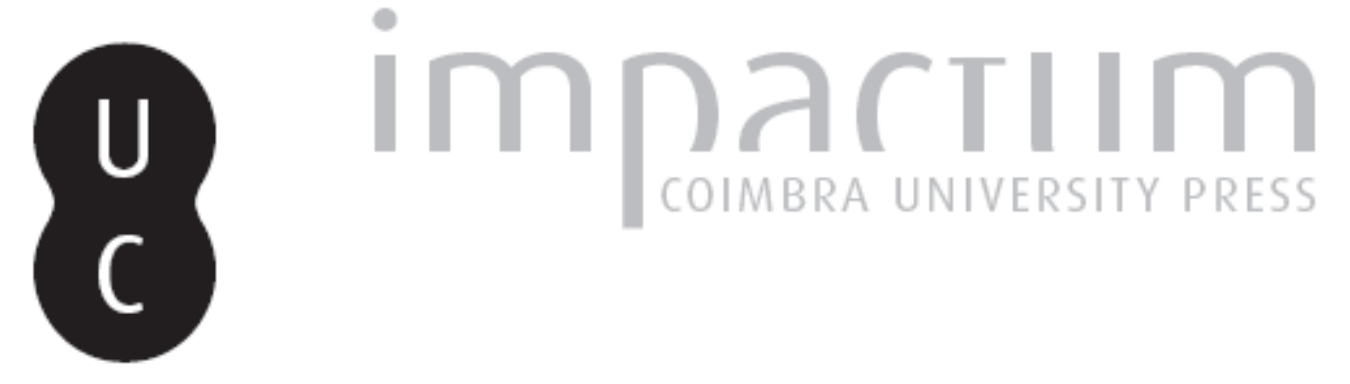

\title{
Subvertir le désir de royaume: enjeux politiques de la déconstruction
}

\section{Autor(es): Weber, Elisabeth}
Publicado por: Faculdade de Letras da Universidade de Coimbra, Instituto de Estudos Filosóficos

URL persistente:

URl:http://hdl.handle.net/10316.2/29463

DOI:

DOI:http://dx.doi.org/10.14195/0872-0851_41_3

Accessed : $\quad$ 26-Apr-2023 12:20:10

A navegação consulta e descarregamento dos títulos inseridos nas Bibliotecas Digitais UC Digitalis, UC Pombalina e UC Impactum, pressupõem a aceitação plena e sem reservas dos Termos e Condições de Uso destas Bibliotecas Digitais, disponíveis em https://digitalis.uc.pt/pt-pt/termos.

Conforme exposto nos referidos Termos e Condições de Uso, o descarregamento de títulos de acesso restrito requer uma licença válida de autorização devendo o utilizador aceder ao(s) documento(s) a partir de um endereço de IP da instituição detentora da supramencionada licença.

Ao utilizador é apenas permitido o descarregamento para uso pessoal, pelo que o emprego do(s) título(s) descarregado(s) para outro fim, designadamente comercial, carece de autorização do respetivo autor ou editor da obra.

Na medida em que todas as obras da UC Digitalis se encontram protegidas pelo Código do Direito de Autor e Direitos Conexos e demais legislação aplicável, toda a cópia, parcial ou total, deste documento, nos casos em que é legalmente admitida, deverá conter ou fazer-se acompanhar por este aviso.

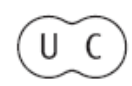




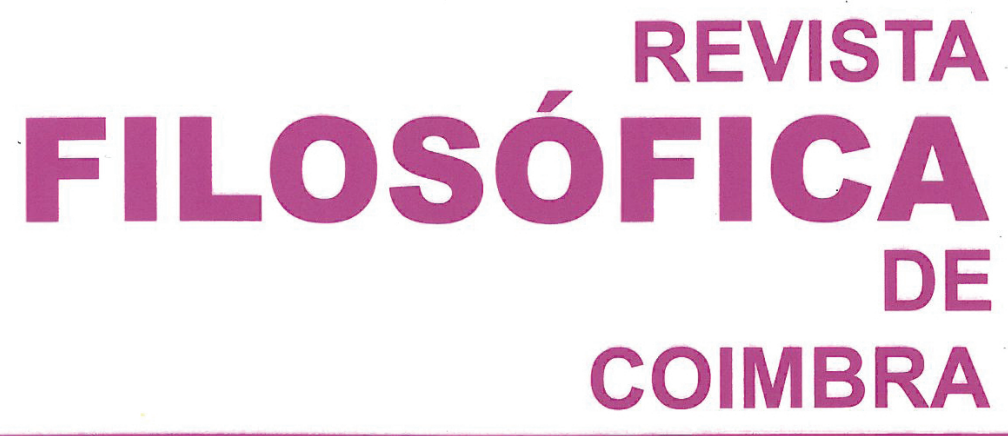

vol. 21 - número 41 - março 2012

vol. 21 - número 41 - março 2012

Fundação Eng. António de Almeida

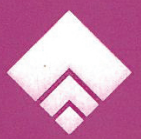




\title{
SUBVERTIR LE DÉSIR DE ROYAUME: ENJEUX POLITIQUES DE LA DÉCONSTRUCTION*
}

\author{
ELISABETH WEBER **
}

\begin{abstract}
Resumo: Este texto (que preserva os traços da cena de seminário em que foi apresentado) oferece uma interpretação do filme de 1990 de Manoel de Oliveira, NON ou a vã glória de mandar, com a ajuda de vários ensaios de Jacques Derrida, incluindo um dos primeiros - o decisivo "La difference». Persegue uma reflexão acerca do que Derrida chama "desejo" do "reino", desconstruindo estratégias para responder e para perscrutar esse desejo - um desejo intimamente ligado à "glória de mandar". Através da interpretação do filme de Oliveira e do trabalho de Derrida, o texto persegue também uma reflexão sobre a crescente legitimidade da tortura, nos Estados Unidos nomeadamente. Inspirado por um ensaio tardio de Derrida que se interroga acerca de «como viver juntos», a última parte do nosso texto apresenta uma leitura de uma das histórias de Franz Kafka que oferece um modelo de comunidade que perscruta igualmente o "desejo do reino" e a "glória de mandar".
\end{abstract}

Palavras Chave: Desconstrução, Democracia, Reino, Comunidade, Perdão, Guerra ao Terror.

Summary: This text (which preserves the traces of the seminar setting where it was presented) offers an interpretation of Manoel de Oliveira's 1990 film NON ou a vã glória de mandar (NON or the vain glory of command) with the help of several of Jacques Derrida's essays, including one of the earliest, the 'landmark' essay «La différance». It pursues a reflection on what Derrida calls the "desire" of "kingdom", and on deconstruction's strategies to respond to and undermine this desire, a desire closely linked to the "glory of command." Through the interpretation of de Oliveira's film and Derrida's work, the text also pursues a reflection on the growing legitimacy of torture, in particular in the United States. Inspired

* Séminaire à l'Université de Coimbra (le 2 décembre 2008).

* University of California, Santa Barbara (USA). 
by a later essay of Derrida's that asks the question of "how to live together," the last part of the text presents a reading of one of Franz Kafka's stories which offers a model of community that equally undermines the "desire of kingdom" and the "glory of command."

Key words: Deconstruction, Democracy, Kingdom, Community, Forgiveness, War on terror.

Tout d'abord, j'aimerais exprimer ma gratitude à Madame le Professeur Fernanda Bernardo pour m'avoir invitée à son séminaire, dans cette université de grand renom et ce si beau pays. Muito obrigada!

Hier, le $1^{\text {er }}$ décembre, votre pays a commémoré le jour de la Restauration, c'est-à-dire le jour où le mouvement patriotique a rétabli l'indépendance du Portugal en installant la dynastie de Bragança sur le trône. La proclamation du roi, le $1^{\text {er }}$ décembre 1640 , en la personne de D. João IV, mit fin à soixante ans de domination espagnole sur le Portugal, une période qui en fait avait débuté deux ans plus tôt, avec la désastreuse bataille d'Alcacer-Quibir et la disparition du roi D. Sebastião, au cours d'une " croisade manquée en quête d'expansion impériale ${ }^{1}$ ".

Permettez-moi donc d'ouvrir mon exposé sur les enjeux politiques de la déconstruction avec un film portugais, qui se termine par une méditation extraordinaire sur la bataille d'Alcacer-Quibir, la célèbre bataille des trois rois. Il s'agit de NON ou a vã glória de mandar, réalisé par Manoel de Oliveira en 1990.

Le titre de ce film n'utilise pas le mot portugais pour " non », não, mais le mot latin, $N O N$, écrit en lettres capitales. C'est un choix significatif. Le « NON » est une citation d'un sermon de 1670 du père António Vieira, qui cite à son tour un autre texte, la traduction latine de l'évangile de Matthieu (Matt. XX, 23). La phrase du Padre A. Vieira citée par de Oliveira est la suivante : "Terrível palavra é um NON » (Terrible parole est un NON).

Des sermons du Padre A. Vieira nul n'a été écrit en latin. Dans le sermon de 1670, le «NON» est le seul mot qui soit latin, à part les citations du nouveau testament et de la traduction latine de la Bible hébraïque.

A l'occasion d'une rencontre sur l'île de Capri, dont le thème était « la religion », Jacques Derrida donna une conférence intitulée « Foi et savoir », dans laquelle il soulignait le rôle décisif de la langue latine: « [...] "la question de la religio" ne se confond-elle pas, tout simplement, si on

\footnotetext{
${ }^{1}$ Randall Johnson, Manoel de Oliveira, Urbana, University of Illinois Press, 2007, p. 64.
} 
peut dire, avec la question du latin ? Par où il conviendrait d'entendre, au-delà d'une "question de langue et de culture", l'étrange phénomène de la latinité et de sa mondialisation. Ne parlons pas ici d'universalité, voire d'une idée de l'universalité, seulement d'un processus d'universalisation finie mais énigmatique. On l'interroge rarement dans sa portée géopolitique et éthico-juridique, là où précisément une telle puissance se trouve relayée, déployée, relancée dans son héritage paradoxal par l'hégémonie mondiale et encore irrésistible d'une "langue", c'est-à-dire aussi d'une culture pour une part non latine, l'anglo-américain. Pour tout ce qui touche en particulier la religion, pour ce qui parle "religion", pour ce qui tient un discours religieux ou sur la religion, l'anglo-américain reste latin. Religion circule dans le monde, on peut le dire, comme un mot anglais qui aurait fait une station à Rome et un détour par les États-Unis. Bien au-delà de ses figures strictement capitalistiques ou politico-militaires, une appropriation hyper-impérialiste est en cours depuis des siècles. Elle s'impose de façon particulièrement sensible dans l'appareil conceptuel du droit international et de la rhétorique politique mondiale. Partout où ce dispositif domine, il s'articule à un discours sur la religion ${ }^{2}$. »

Derrida affirme donc que l'appareil conceptuel du droit international et de la politique mondiale est aujourd'hui marqué par une « appropriation hyper-impérialiste » qui est « en cours depuis des siècles ». Par conséquent, nous sommes, aujourd'hui, au Portugal comme aux États-Unis, les héritiers de cette « appropriation ».

L'hypothèse de Derrida, c'est que la mondialisation (appelée « globalization » en anglo-américain ${ }^{3}$ ) est anglo-américaine, et que l'anglo-américain est, en son fond, et surtout quand il s'agit de « religion », latin. C'est pourquoi Derrida a créé, comme vous savez, le concept de mondialatinisation.

À la même page de « Foi et savoir », Derrida précise que « "mondialatinisation" (essentiellement chrétienne, bien sûr) [...] nomme un événement unique au regard duquel un métalangage paraît inaccessible, alors qu'il reste ici, pourtant, de première nécessité ».

Les langues du monde, et les langues des institutions du monde, y compris le discours universitaire, sont si étroitement et si profondément impliquées dans cet «événement » qu'elles ne sont pas encore capables d'inventer un métalangage, si « nécessaire » soit-il. C'est donc une tâche,

2 Jacques Derrida, «Foi et savoir», dans La Religion, sous la direction de Jacques Derrida et Gianni Vattimo, Paris, Seuil, 1996, p. 42.

${ }^{3}$ Dans L'Université sans condition (Paris, Galilée, 2001), Derrida précise qu'il « garde le mot français de "mondialisation" pour "globalization" ou "Globalisierung" afin de maintenir la référence à un "monde" (world, Welt, mundus) qui n'est ni le globe, ni le cosmos, ni l'univers ». 
une tâche qu'il reste à accomplir. Et l'une des « inventions de l'autre ${ }^{4}$ » qui est urgente aujourd'hui.

Voici qui est cependant clair : le latin et la mondialatinisation sont inséparables de « l'appropriation hyper-impérialiste » dont parle Derrida, donc d'un certain empire, de la domination de l'empire. Il n'y a guère de doute que de nos jours aucun autre pays n'incarne " l'empire » plus que les États-Unis.

Pour une approche qui s'inspire de la déconstruction, un seul mot, en l'occurrence le «NON» du titre du film réalisé par de Oliveira, suffit à nous conduire au cœur de questions d'une importance politique aiguë. Et bien que nous nous rencontrions aujourd'hui dans une langue qui n'est ni la vôtre ni la mienne ${ }^{5}$, nous pouvons être assurés (bien que tout sauf rassurés) que nous partageons, à travers nos institutions respectives, le latin angloaméricanisé. Voilà une des intrications que Derrida nous invite à penser.

C'est un exemple des enjeux politiques de la pratique qu'est la déconstruction : comme Derrida l'a précisé à plusieurs reprises, elle n'existe qu'au pluriel. Il n'y a pas LA déconstruction, il y a seulement des déconstructions, toujours plurielles, toujours au pluriel. C'est là une invitation à une pratique, une invitation à quitter une pensée qui scinderait la théorie et la pratique, pour ne citer que cette vieille opposition.

Revenons un instant au film de Manoel de Oliveira. Le titre fait de la gloire de « commander» un équivalent du «NON» ${ }^{6}$, et ce «NON», comme dit le chevalier portugais en s'adressant au spectateur dans son monologue sur le «non », dix minutes avant la fin du film, tue l'espoir.

Je propose que nous regardions deux courts extraits du film de Manoel de Oliveira ${ }^{7}$. Le premier montre un groupe de soldats portugais en train de manger à la veille d'une expédition de reconnaissance dans une des colonies portugaises en Afrique, la Guinée portugaise. L'officier du groupe, le lieutenant Cabrita, était étudiant en histoire avant la guerre, et il parle à ses camarades de l'histoire du Portugal à travers ses batailles militaires décisives. Dans l'extrait que nous allons voir, le lieutenant Cabrita discute avec ses camarades sur le mythe du Cinquième Empire.

Le deuxième extrait traite de la fameuse bataille d'Alcacer-Quibir et se termine par un monologue saisissant sur le «NON», qui est prononcé

\footnotetext{
${ }^{4}$ Cf. Jacques Derrida, Psyché. Inventions de l'autre, Paris, Galilée, 1987.

${ }^{5}$ Le séminaire fut conduit en français.

${ }^{6} \mathrm{Le}$ « ou » du titre a la valeur logique d'une conjonction coordonnante, d'un « ou » inclusif, pour reprendre les termes de la logique formelle.

${ }^{7}$ Le premier extrait: de la 62e à la $67 \mathrm{e}$ minute, le deuxième extrait: de la 92e à la 103 e minute.
} 
par un noble portugais : "Enrobez tant que vous voudrez le NON, il sera toujours amer, Dorez-le autant que vous voulez, il sera toujours en fer ! »

Ce monologue est en fait, comme je l'ai dit, une citation d'un sermon du père António Vieira prononcé en 1670, donc quatre-vingt-douze ans

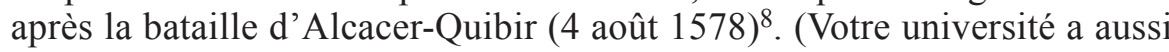
célébré récemment le quatrième centenaire de la naissance du P. A. Vieira. Il est notoire que A. Vieira fut exilé dans votre ville en 1663, et condamné ici aussi par le tribunal de l'Inquisition en 1667.)

Vers la fin du film, achevant son monologue, le chevalier portugais se suicide avec le fer de son épée, et il s'effondre, mourant, sur le drapeau de saint Georges, le saint patron du Portugal. Saint Georges est le plus célèbre des « saints militaires » ou " saints soldats» : plus qu'aucun autre des saints patrons, il incarne l'impossibilité de séparer la religion et l'empire.

Comme j'espère le montrer par la suite, le film de Manoel de Oliveira suggère que l'impérialisme comme la religion, tous deux essentiellement latins, sont inséparables de ce que Derrida appelait, dans son texte « La différance », donc déjà en 1968, le désir de royaume ${ }^{9}$. Dans le film, l'expression de ce désir de royaume culmine dans le « NON», cette « terrible parole ».

Comme vous le savez sans doute, le sermon du Padre António Vieira fut prononcé dans la chapelle royale, "na Capela Real ", devant la cour du régent D. Pedro (qui fut proclamé roi en 1683, Don Pedro II, mais qui régnait depuis 1668). Le but du sermon était de décourager les notables, les courtisans, tous ceux qui avaient des requêtes, dans leur volonté d'approcher le roi avec des demandes qui l'auraient forcé à répondre par un «non ».

Pourquoi le «NON» est-il «terrible »? Padre A. Vieira en donne plusieurs raisons: "Terrivel palavra é um NON. Não tem direito nem avesso : por qualquer lado que o tomeis, sempre soa e diz o mesmo. Lede-o do princípio para o fim, ou do fim para o princípio, sempre é Non. Quando a vara de Moisés se converteu naquela serpente tão feroz, que fugia dela porque o não mordesse; disse-lhe Deus que a tomasse ao revés, e logo perdeu a figura, a ferocidade e a peçonha. $O$ Non não é assim : por qualquer parte que tomeis sempre é serpente, sempre morde, sempre fere, sempre leva o veneno consigo. Mata a esperaça, que é o último remédio que deixou a natureza a todos os males. Não há corretivo que o modere, nem arte que o abrande, nem lisonja que o adoce. Por mais que o confeiteis um não sempre amarga; por mais que o enfeiteis sempre é feio ; por mais que o doureis sempre é de ferro. Em nenhuma

${ }^{8}$ Et environ deux ans après le traité de paix avec l'Espagne, qui conclut trente ans de guerre.

9 Jacques Derrida, Marges de la philosophie, Paris, Minuit, 1972, p. 22 
solfa o podeis pôr que não seja mal soante, áspero e duro. Quereis saber qual é a dureza de um não ? A mais dura coisa que tem a vida é chegar a pedir e depois de chegar a pedir ouvir um não : vede o que será? A lingua Hebraica, que é a que falou Adão, e a que mais naturalmente significa e declara a essência das coisas chama ao negar o que se pede, envergonhar a face. Assim disse Betsabé a Salomão : Petitionem unam precor a te, ne confundas faciem neam ${ }^{10}$ : trago-vos Senhor, uma petição, que não me envergonheis a face. E por que se chama envergonhar a face negar o que se pede ? Porque dizer não a quem pede, é dar-lhe uma bofetada com a lingua. Tão dura, tão áspera, tão injuirosa palavra é um não. Para a necessidade dura, para a honra afrontosa, e para o merecimento insofrivel ${ }^{11}$. »

Pour le P. António Vieira, le «NON», mais aussi le «não », est une gifle, qui serait donnée par la langue. Le « NON» fait monter la « honte au visage » (comme on dit " rougir de honte »), et le but du sermon est d'exhorter les nobles et les proches du roi à ne pas mettre le roi dans une situation où il serait forcé de rendre honteux ses sujets.

Il est tout à fait significatif que, citant la Bible hébraïque, le Padre A. Vieira identifie le «NON» et le «não » avec quelque chose qui rend honteux, plus spécifiquement qui fait monter la « honte au visage ». Car la honte naît de la négation non pas seulement de ce qui est demandé, mais de l'existence de celui ou celle qui demande. C'est d'ailleurs, soit dit en passant, une des caractéristiques les plus dévastatrices de la torture. Comme en ont témoigné maintes fois les ex-détenus à Abou Ghraïb, les tortures et les humiliations ont été vécues par eux comme visant à infliger une honte ineffaçable à la victime ${ }^{12}$. Cette honte revient à rien de moins qu'à l'annihilation. La honte est annihilante. Pour le chevalier portugais du film de Manoel de Oliveira, citant le Padre A. Vieira, le « NON » anéantit.

101 Romains, II, 16 [Assim, que agora uma só petição te faço; não ma rejeites. E ela lhe disse : Fala.]

${ }^{11}$ Padre António Vieira, Sermões, t. I, Organização Alcir Pécora, São Paulo, Hedra, 2003, p. 250

${ }^{12}$ Un Iraqien interviewé par le journaliste américan Mark Danner, à Fallouja, parle de l'humiliation de l'occupation et de la honte de la torture : Mark Danner, "Torture and Truth. Amercia, Abu Ghraib, and the War on Terror ", New York Review of Books, 2004, p. 1. On pense aussi à la fameuse phrase de Jean Améry : "Celui qui a été soumis à la torture est désormais incapable de se sentir chez soi dans le monde [heimisch sein in der Welt]. L'outrage [Die Schmach; aussi 'la honte'] de l'anéantissement est indélébile. » Jean Améry, Par-delà le crime et le châtiment. Essai pour surmonter l'insurmontable, trad. de 1'allemand par Françoise Wuilmart, Arles: Actes Sud 1995, p. 79. 
J'ai commencé notre conversation d'aujourd'hui avec ce film, parce qu'il me semble avoir de fortes affinités avec quelques thèmes " fondamentaux », si je puis dire, de la pensée de Derrida, et de la déconstruction.

Pour répondre au « non » de fer, au « non » de la gloire de commander, au «non » de la gloire, au « non » du commander, au « non » qui tue, Derrida choisit de ne pas simplement lui opposer un « oui ». Ce serait naïf et voué à l'échec. Pour lui, la tâche est de dégager, dans le « NON », non pas le " oui » d'une affirmation, aboutissant en fin de compte à une Aufhebung dialectique à la Hegel, mais un " oui » promettant toujours d'autres « oui », un " oui » qui d'emblée, de façon an-archique, immémoriale et multiple, disséminante, accueille la différence de l'autre, la parole de l'autre. Pour ce « oui », la parole de l'autre est celle d'une " commande » sans doute, mais commande d'une autre nature. Une des tâches de la déconstruction est précisément de dégager, dans la gloire de commander, l'autre, peut-être l'ennemi, qui s'y est insinué depuis toujours, qui l'habite en secret et qui, en fait, depuis un for intérieur radicalement décentré, la commande.

Pour mieux comprendre cette dynamique, prenons quelques lignes de Voyous ${ }^{13}$ : «La pensée du politique a toujours été une pensée de la différance, et la pensée de la différance toujours aussi une pensée $d u$ politique. » Et : « Toute trace est trace de démocratie. »

Puisque Derrida se réfère explicitement, dans ces lignes de 2002, à la différance, il m'a paru utile de relire avec vous le texte de 1968, avec lequel Derrida ébranla le monde philosophique français et, par la suite, international, et qui a pour titre $«$ La différance ${ }^{14} »$. Derrida commence ce texte en notant qu'il a introduit la « lettre première ", "s'il faut en croire l'alphabet » dans l'écriture du mot différence, une introduction qui aura comme résultat de complètement subvertir la valeur de tout «premier ». Cela aura des conséquences sans fin : si l'on subvertit la valeur de " premier », toutes les hiérarchies sont menacées, voire, précisément, subverties.

À la première page, Derrida nous donne d'autres indications précieuses : " On pourra toujours faire comme si cela ne faisait pas de différence. » Et ce néo-graphisme s'est imposé : « j'ai pu [...] me laisser imposer » le concept de différance, un concept qui en fait n'est pas un.

Le geste est radical, surtout dans le monde philosophique académique, où le discours est traditionnellement une affaire de maîtrise, de contrôle, et sans doute de position aux commandes, surtout si l'on tient compte

\footnotetext{
13 Jacques Derrida, Voyous, Paris, Galilée, 2993, p. 64.

14 « La différance », dans Marges de la philosophie, p. 3 et suiv.
} 
$\mathrm{du}$ « lien entre les héritages philosophiques et la structure du système juridico-politique encore dominant ${ }^{15} »$.

Voilà un philosophe qui se laisse dicter un " manquement silencieux à l'orthographe ${ }^{16}$ ", qui se laisse, donc, détourner, en s'engageant dans un chemin qui ne suit pas la graphie " propre », le contrôle, la maîtrise, l'autorité traditionnellement associés au discours philosophique.

Le fait que l'on " pourra toujours faire comme si cela ne faisait pas de différence ${ }^{17}$ » confirme qu'il s'agit d'un détournement aussi discret que radical. Il est discret parce que l'autre habite déjà le même, l'a habité depuis toujours, et ce que la déconstruction entreprend, c'est de suivre et d'aggraver les fissures dans l'édifice du même. Ou, plutôt que les aggraver, de les célébrer et de les accueillir par un " oui, oui » toujours multiple, car tout « oui » est déjà, selon Derrida, la promesse d'autres affirmations à venir.

La nature discrète, presque imperceptible de la différance, est explicitée plus loin dans le texte : la « différance n'est pas. Elle n'est pas un étant-présent, si excellent, unique, principiel ou transcendant qu'on le désire. Elle ne commande rien, ne règne sur rien et n'exerce nulle part aucune autorité. Elle ne s'annonce par aucune majuscule. Non seulement il n'y a pas de royaume de la différance mais celle-ci fomente la subversion de tout royaume. Ce qui la rend évidemment menaçante et infailliblement redoutée par tout ce qui en nous désire le royaume, la présence passée ou à venir d'un royaume ${ }^{18} »$.

Il ne s'agit pas seulement de développer un discours qui critiquerait et déconstruirait l'autorité, la souveraineté, la gloire de commander. Car, en fin de compte, il est relativement facile de critiquer ou de dénoncer l'autorité de l'autre. Ce qui est, potentiellement, beaucoup plus difficile, c'est d'engager une réflexion et des déconstructions de « tout ce qui en nous désire le royaume ${ }^{19} »$, de tout ce qui en nous désire participer à la gloire de commander. Car, ce qui se sent à juste titre menacé par « la différance », ce qui la redoute « infailliblement » et terriblement, c'est « ce qui en nous désire le royaume ».

Pour explorer les implications de cette thèse, revenons au film de Manoel de Oliveira. Au cours du premier extrait, les soldats parlent du Cinquième Empire qui, comme le dit l'officier Cabrita, « devait dominer le monde avec un seul roi, un seul pape. Le P. A. Vieira en parlait

15 Jacques Derrida, « Auto-immunités, suicides réels et symboliques », dans Jacques Derrida, Jürgen Habermas, Le "Concept » du 11 septembre. Dialogues à New York (octobre-décembre 2001) avec Giovanna Borradori, Paris, Galilée, 2003, p. 161.

16 « La différance », art. cité, p. 3.

17 Ibid., p. 3.

18 « La différance », art. cité, p. 22. Je souligne (E.W).

19 Je souligne $(\mathrm{EW})$. 
comme l'instauration du règne du Christ sur terre, un règne de mille ans, jusqu'à la venue de l'Antéchrist. Un règne universel englobant tout : continents, races, cultures ${ }^{20} »$. En bref, le Cinquième Empire rêvé par le P. A. Vieira, inspiré par le messianisme du cordonnier Bandarra, envisageait un ensemble universel, englobant tout, véritablement catholique.

L'officier Cabrita décrit le Cinquième Empire comme une utopie « qui s'oppose au désir de dominer le monde par la force du pouvoir », un « désir latent » qu'il voit à l'œuvre « dans l'ambition des grandes puissances comme la Russie ou l'Amérique » qui voudraient instaurer " une sorte de Cinquième Empire par la force "».

Toute la pensée de Derrida nous enseigne qu'une telle opposition entre un Cinquième Empire de paix universelle et un Cinquième Empire «par la force » s'effrite sous un regard critique et déconstructeur aussi sûrement que les oppositions métaphysiques. Et tout le film de Manoel de Oliveira démasque la chimère d'une telle opposition, dès la première minute qui plonge le spectateur dans un espace noir percé seulement par la musique endeuillée d'un trio à cordes, et, après une vingtaine de longues secondes, la citation du P. A. Vieira, "Terrível palavra é um NON », jusqu'aux dernières minutes, qui obligent le spectateur à être au plus près le témoin des dernières minutes de la vie de l'officier Cabrita, de son arrêt cardiaque et des vains efforts des médecins militaires pour le ressusciter.

Le langage filmique est éloquent sur le fait que les deux versions du Cinquième Empire sont une seule et même version. Non seulement « le plus grand désastre de notre histoire » a donné naissance au fantasme du Cinquième Empire, comme l'explique l'officier Cabrita, et ce « désastre [est] devenu mythe », précise son camarade, le soldat Manoel, à quoi le lieutenant Cabrita ajoute : "Mythe devenu vérité. » Mais l'inverse n'a, sans doute, pas moins de " vérité », à savoir que ce qui s'annonce au nom de l'utopie, d'une paix universelle, est poursuivi à travers les plus grands désastres de notre histoire, sinon par leur truchement.

Si cette problématique peut paraître plutôt évidente dans le cas des " Empires » historiques ou actuels, Derrida nous invite à y voir aussi et peut-être même surtout une des grandes épreuves d'une pensée de la démocratie. Derrida nous enjoint de penser la « cratie » dans démocratie, autrement dit la source et l'emploi de la « force » dans la " démocratie », la gloire de commander, le désir de royaume au cœur des institutions démocratiques.

Pour prendre un exemple douloureux et très actuel, considérons le fait que la démocratie la plus puissante du monde, la démocratie qui se proclame régulièrement la «meilleure » du monde, les États-Unis,

${ }^{20} 62-63^{\mathrm{e}}$ minutes du film. 
a énoncé officiellement, à l'automne de 2006, une loi autorisant la torture, le «Military Commissions Act, 2006 », même si, évidemment, le mot «torture » n'apparaissait pas dans le texte. De plus, l'article $7 \mathrm{du}$ Military Commissions Act éliminait le droit du « Habeas Corpus » pour les personnes suspectes d'activités ou de sympathies terroristes, autrement dit le droit pour tout détenu d'apprendre dans une cour la raison de son incarcération. Le 12 juin 2008, la Cour suprême a déclaré l'article 7 inconstitutionnel.

Le 22 janvier 2009, deux jours après son investiture, le président Barack Obama ordonna, par une série de décrets, aux forces armées et au personnel de la CIA (Central Intelligence Agency) de respecter la prohibition de la torture, telle qu'elle est spécifiée dans le manuel pratique de l'armée ${ }^{21}$. Cependant, l'administration Obama refuse jusqu'aujourd'hui le droit du « Habeas Corpus » à ces détenus qui languissent depuis des mois, voire des années, dans les prisons des bases militaires américaines, telle celle de Bagram, en Afghanistan. De plus, il est impossible de vérifier si les prisons clandestines de la CIA, les black sites (" sites noirs »), inaccessibles aux représentants d'organisations humanitaires comme la Croix Rouge, ainsi qu'aux avocats et familles des détenus, ont bien été fermées, comme Obama le promit le 22 janvier $^{22}$.

${ }^{21}$ Voir : http://www.courrierinternational.com/article/2009/01/23/obama-met-presquefin-a-la-torture

22 Voir : http://internationalnews.over-blog.com/article-30231224.html. Paragraphe ajouté en juillet 2009, lors de la rédaction du séminaire pour la publication. Le 13 septembre 2009, le gouvernement Obama a annoncé qu'il publierait de nouvelles directives pour permettre aux détenus de Bagram de contester leur détention. Un article publié sur le site Internet de la « American Civil Liberties Union (ACLU) » dit : « The guidelines would reportedly assign military officials to detainees to gather information about the circumstances of their detention, which could then be used to challenge their detention before a military-appointed review board. » Une des avocates de la ACLU, Melissa Goodman, est cité : «The Defense Department's commitment to give prisoners held at Bagram more procedural rights is encouraging, especially since some of the prisoners held there have now been imprisoned for six years without access to lawyers, without charge or trial, and without any meaningful opportunity to challenge their detention. We look forward to seeing the details of the new policy; until then, it is difficult to assess how meaningful a change it represents. We remain concerned, however, about the level of secrecy that surrounds Bagram. The public remains uninformed of basic facts such as who is imprisoned there, how long they have been held, where they were captured and on what grounds they are being subjected to indefinite detention. The government should make public documents that could shed light on this crucial information about the detention and treatment of prisoners at Bagram. We also remain deeply concerned that Bagram is being used not only as a battlefield detention facility but as a prison to hold terrorism suspects apprehended all over the world. While moves to give prisoners a better chance to contest their prolonged 
Sur cet arrière-fond actuel, il importe d'avoir présent à l'esprit comme Darius Rejali, auteur de Torture and Democracy ${ }^{23}$, l'a montré avec autorité, que ce sont précisément les gouvernements démocratiques qui utilisent des méthodes de torture invisibles, indécelables. Si les dictatures pratiquent la torture pour terrifier les populations, usant de méthodes qui laissent des traces visibles, les démocraties, elles, essaient de se soustraire à la vigilance des organisations des droits de l'homme. L'argument fréquemment avancé dans l'opinion publique, selon lequel démocratie et torture s'excluent mutuellement, ne fait que consolider l'opinion naïve qui ne voit dans l'usage de la torture par un régime démocratique rien qu' une aberration.

La question que l'œuvre de Derrida nous aide à développer est la suivante : qu'y a-t-il dans le cœur même de la démocratie qui permet, appelle, requiert, voire désire l'usage de la torture ? Voilà un exemple, et un exemple des plus cruciaux, pour penser la "force ", la «cratie » dans démocratie, la violence qui n'est pas l'autre de la démocratie, mais est produite, pour citer Derrida, comme une réaction « auto-immunitaire ».

Dans ses grands livres Politiques de l'amitié et Voyous, Derrida nous enjoint de penser aussi la première partie du mot "démocratie »- le demos. Puisque notre temps est limité, je soulignerai seulement un aspect de cette pensée : la question du demos, du peuple, comme « ensemble ».

Comme nous l'avons vu dans les paroles du lieutenant Cabrita, le fantasme du Cinquième Empire peut être décrit comme le fantasme d'un ensemble universel. Cabrita explique que ce fantasme est issu du «plus grand désastre de notre histoire », un " désastre devenu mythe ", comme son camarade, le soldat Manoel, le précise, à quoi Cabrita ajoute : « Mythe devenu vérité. » Et il cite une phrase qui semble inspirée d'un mystique portugais du $\mathrm{XVI}^{\mathrm{e}}$ siècle, le cordonnier Bandarra, dont la vision du « $\mathrm{O}$ Encoberto », du roi et sauveur « caché », a connu une popularité énorme à travers les siècles, jusqu'au célèbre « Mensagem » de Fernando Pessoa :

detention in a military forum are encouraging, the Obama administration's continued efforts to block Bagram prisoners' access to U.S. courts remains disappointing." Voir: http://aclu.org/safefree/detention/40982prs20090913.html. (Addendum février 2012 : Le 31 décembre 2011, Obama signa « l'acte d'autorisation de défense nationale » (National Defense Authorization Act, NDAA). Cette loi permet aux forces militaires et de sécurité américaines d'arrêter, partout dans le monde, y compris à l'intérieur des Etats-Unis, les personnes soupçonnées de «terrorisme » ou de sympathiser avec des terroristes, et de les emprisonner, sans, procès juridique, pour une durée indéterminée.)

${ }^{23}$ Darius Rejali, Torture and Democracy, Princeton et Oxford, Princeton University Press, 2007 (880 pages). 
«Vérité ! Secrète et inexplicable. Au lieu d'un sens logique, cette vérité a un sens dernier qui explique tout ${ }^{24}$. »

Ce sont ces mêmes vers que Cabrita citera de nouveau vers la fin $\mathrm{du}$ film ${ }^{25}$, dans son délire où il revit le désastre de la bataille d'AlcacerQuibir, délire qui se termine par le terrifiant monologue sur le «NON».

La conversation entre Cabrita et Manoel est extrêmement perspicace : ce qui est clair pour le fantasme du Cinquième Empire vaut peut-être pour les empires en général. Leurs fondements sont des désastres transformés en mythe et en mystique : autre version du « fondement mystique de l'autorité $^{26}$ ». Que la « vérité » soit fondée sur le mythe, cela a, évidemment, perturbé la philosophie dès sa «fondation » chez Platon.

Dans son délire, Cabrita lie étroitement le Cinquième Empire au « NON », l'utopie d'un ensemble où règne la paix universelle à la destruction engendrée par la gloire de commander. L'utopie du Cinquième Empire est une expression évidente de ce qui, pour revenir à Derrida, " en nous désire le royaume ». Le film ne laisse aucune illusion sur les conséquences de ce désir.

Évidemment, je cite ces passages aussi parce que le pays dans lequel je vis, les États-Unis, est engagé dans deux guerres, au nom de ce que l'on appelle "démocratie " et qui, à en croire le discours américain officiel, s'opposerait (je cite encore le lieutenant Cabrita) « au désir de dominer le monde par la force du pouvoir ». Or ce que Cabrita appelle le " désir latent » dans « l'ambition des grandes puissances comme [...] l'Amérique » qui voudraient instaurer « une sorte de Cinquième Empire par la force », ce désir latent n'est que trop apparent, dans la politique étrangère des ÉtatsUnis. Le film de Manoel de Oliveira résonne fortement avec le contexte politique américain actuel, y compris avec ce que l'on appelle, aux ÉtatsUnis, « la guerre contre le terrorisme ». Soit dit en passant, l'expédition militaire dans une des colonies africaines est aussi décrite, dans le film, comme nécessaire pour combattre les « terroristes » $\left(55^{\mathrm{e}}\right.$ minute) .

Mais le film résonne aussi à cause de sa critique subtile et explicite du messianisme politique. Comme Derrida l'écrit en maintes occasions, ce qui « en nous désire le royaume » est aussi ce qui en nous désire le " sauvetage », la rédemption, l'indemnisation, le devenir-entier (pour citer quelques-unes des expressions de « Foi et savoir »), l'entrée dans la terre promise. À cet égard, la multitude de chansons écrites avant les élections américaines de novembre 2008 pour Barack Obama sont révélatrices, en

${ }^{24} 65^{\mathrm{e}}$ minute du film. Bien que la phrase soit présentée comme une citation, elle est, en fait, de Manoel de Oliveira (communication orale).

${ }^{25}$ Ces mêmes mots sont déjà cités par Cabrita plus tôt dans le film, pendant le voyage des soldats dans le camion militaire ( $40^{\mathrm{e}}$ minute).

26 Voir Jacques Derrida, Force de loi, Paris, Galilée, 2005. 
particulier l' « American Prayer ${ }^{27}$ » de Dave Stewart, qui a l'audace de citer le serment que Martin Luther King Jr. prononça la veille de son assassinat, le 3 avril 1968, dans la ville de Memphis, au Tennessee. Au cours de ce serment, King dit avoir vu la terre promise : "I may not get there with you [...] but I want you to know, tonight ["Yes!'”] that we as a people will get to the promised land ${ }^{28 !} \gg$ Dans le vidéo-clip accompagnant la chanson, Barack Obama est évoqué comme le messie qui nous guidera là-bas.

Dans les traditions de l'Occident, le désir de royaume a souvent été inséparable d'un désir de roi-sauveur, de messie. Dans votre pays, le mythe du roi caché a donné lieu à toute une tradition d'expressions puissantes à ce désir. Si désirable, et par beaucoup désirée, que l'élection de Barack Obama ait été, il ne sera possible de dépasser la phase de "l'espoir » sur lequel le nouveau président a bâti sa candidature qu'à condition de contrecarrer activement les tendances de son idéalisation et de sa «messianisation».

Revenons au texte de 1968, "La différance ».

J'ai déjà évoqué l'aveu de Derrida selon lequel il s'est laissé « imposer » le manquement à l'orthographe dans le mot ou le concept de différance. Il s'agit, donc, d'un certain renoncement à la maîtrise philosophique. Quelques pages plus loin, Derrida revient à la question de la « maîtrise » en spécifiant que « la différance, qui n'est ni un mot ni un concept, m'a paru stratégiquement le plus propre à penser, sinon à maîtriser - la pensée étant peutêtre ici ce qui se tient dans un certain rapport nécessaire avec les limites structurelles de la maîtrise - le plus irréductible de notre "époque" » (p. 7).

Bien que la formulation de Derrida soit prudente, relevant du régime du « peut-être » dont, inspiré de Nietzsche, il donnera, dans Politiques de l'amitié, des analyses inoubliables ${ }^{29}$, je voudrais avancer ici la thèse selon laquelle " le plus irréductible de notre "époque" » ne peut être "pensé » qu'à condition que la pensée " se tien[ne] dans un certain rapport nécessaire avec les limites structurelles de la maîtrise ». C'est pourquoi la différance n'est pas un concept, en aucun cas un concept au sens hégélien. Plutôt, " l'efficace de cette thématique de la différance [...] devra être un jour relevée, se prêter d'elle-même, sinon à son remplacement, du moins à son enchaînement dans une chaîne qu'elle n'aura jamais commandée » - autre preuve qu'elle « n'est pas théologique» (p. 7).

Autrement dit, la différance forme un des multiples maillons d'une

${ }^{27} \mathrm{http}: / /$ www.youtube.com/watch? $\mathrm{v}=\mathrm{oVi} 4 \mathrm{rUzf}-0 \mathrm{Q}$

${ }^{28} \mathrm{Cf}$. la description détaillée et émouvante du serment dans «I have seen the promised land ", Time, Sunday, $1^{\text {er }}$ janvier 2006, http://www.time.com/time/magazine/article/0,9171,1145260-10,00.html (accédé le 15 juillet 2009); l'auteur de l'article n'est pas nommé.

${ }^{29}$ Cf. « Aimer d'amitié : peut-être - le nom et l'adverbe », dans: Jacques Derrida, Politiques de l'amitié, Paris, Galilée, 1994, p. 43 et suiv. 
chaîne de concepts/non-concepts, qui, différents, bien sûr, les uns des autres, restent liés et se laissent échanger par d'autres pour penser « le plus irréductible de notre "époque" ». C'est pourquoi, dans l'œuvre ultérieure de Derrida, la différance a été relayée par d'autres maillons de cette chaîne qu'elle ne commande pas, tels que, entre autres, les concepts qui n'en sont pas de «l'auto-immunité » et de "l'hostipitalité $30_{\text {». }}$.

Je voudrais insister là-dessus. Une des difficultés de cette pensée, c'est qu'elle requiert de nous de reconnaître la pensée que nous avons, bien sûr, appris comme une méthode ou un faisceau de méthodes de maîtrise, de reconnaître cette pensée, donc, comme quelque chose qui se " tient dans un certain rapport nécessaire avec les limites structurelles de la maîtrise ». C'est la condition de penser l'autre, de penser l'altérité. Et c'est, bien sûr, une des apories que la pensée de Derrida n'hésite pas à approfondir toujours de nouveau : car toute pensée ne peut se faire qu'à condition d'une certaine maîtrise.

Comment Derrida articule-t-il ce « rapport nécessaire avec les limites structurelles de la maîtrise » dans ce court texte de 1968 ?

Examinons un des arguments. Différance est écrit avec un $a$ parce que « la terminaison ance reste indécise entre l'actif et le passif » (p. 9). Derrida introduit ici un des thèmes qui seront si importants dans son œuvre ultérieure : celui de l'indécision, du moment suspendu, y compris du moment de suspension au battement du cœur de l'autre ${ }^{31}$. Il note que " ce qui se laisse désigner par "différance" n'est ni simplement actif ni simplement passif, annonçant ou rappelant plutôt quelque chose comme la voix moyenne [...] Or la voix moyenne, une certaine non-transitivité, est peut-être ce que la philosophie, se constituant en cette répression, a commencé par distribuer en voix active et voix passive » (p. 9).

Je souligne : la philosophie s'est donc constituée en cette répression qui a fait disparaître la voix moyenne pour « la distribuer en voix active et voix passive $»$.

Il n'y a pas, dans la grammaire des langues modernes, de voix moyenne. Par conséquent, il est extrêmement difficile, sinon impossible, de penser en français (ou en anglais, allemand ou portugais) ce qu'est « la voix

${ }^{30}$ Cf. Jacques Derrida, «Question d'étranger: venue de l'étranger », Quatrième séance (le 10 janvier 1996), dans: Anne Dufourmantelle invite Jacques Derrida à répondre de l'hospitalité, Paris, Calmann-Lévy, 1997, p. 45, où Derrida parle des « interprétations de Benveniste, notamment [...] deux dérivations latines: l'étranger (hostis) accueilli comme hôte ou comme ennemi. Hospitalité, hostilité, hostipitalité. "

31 Je me permets de renvoyer ici à mon texte « Suspended from the Other's Heartbeat », in South Atlantic Quarterly, numéro spécial sur Jacques Derrida, édité par Ian Balfour et Deborah Esch, 2007, pp. 325-34. 
moyenne »-petite illustration pratique des « limites structurelles de la maîtrise $»$.

Derrida donne deux exemples qui pourraient illustrer la voix moyenne : la résonance n'est pas " l'acte de résonner », et la mouvance ne signifie pas, en français, « le simple fait de mouvoir, de se mouvoir ou d'être $\mathrm{mu} »^{32}$. Plus tard, dans Politiques de l'amitié, Derrida ajoute le beau mot d' " aimance ». Commentant l'Éthique à Eudème d'Aristote, Derrida écrit qu' '" au-delà de toute frontière ultérieure entre l'amour et l'amitié, mais aussi entre la voix passive et la voix active, entre l'aimer ou l'être-aimé, il y va de l'aimance ${ }^{33}$ »).

Peut-être que, pour approcher la voix moyenne, supprimée par la constitution même de la philosophie, la définition du langage par Walter Benjamin nous servira.

Benjamin s'intéresse au langage parce qu'il le considère comme un médium de communication, ou plutôt comme un médium de partage (en allemand : Mitteilung). Le langage se communique, ou plutôt, se par-tage : pour éviter le rapprochement avec les sciences, purement utilitaires, de la communication, je traduirai ensuite Mitteilung par " par-tage ». Le langage se par-tage, et c'est pourquoi tout participe du langage, y compris le vivant non humain et les choses inanimées ${ }^{34}$.

La pensée de Benjamin s'inspire ici fortement du mysticisme de la tradition juive, qui reprend littéralement le récit biblique de la genèse du monde : ce sont les paroles de Dieu qui créèrent le ciel et la terre ainsi que tous les vivants, donc l'instrument et le médium de création est la langue, autrement dit, les vingt-deux lettres de la langue dite originaire, l'alphabet hébraïque. Tout est fait non seulement par le langage, mais le langage est aussi la matière, l'étoffe, pour ainsi dire, de la création ${ }^{35}$.

Pour Benjamin, le langage est un médium au sens où le sujet qui se communique ou se partage (à savoir le langage) « est à l'intérieur du procès ${ }^{36}$ ».

32 « La différance », art. cité, p. 9. Selon le Petit Robert, une des significations de « mouvance » est le caractère de ce qui est mouvant (Petit Robert, 1979, p. 1239).

33 Politiques de l'amitié, op. cit., p. 23.

${ }^{34}$ Cf. Walter Benjamin, "Über Sprache überhaupt und über die Sprache des Menschen”, in Walter Benjamin, Gesammelte Schriften, Band II.1, Frankfurt: Suhrkamp 1980, pp. 140 et suiv.

35 Cf. Barbara Holdrege, Veda and Torah, Albany: State University of New York Press, 1996, pp. 167 et suiv.

${ }^{36}$ Cf. Wolf Kittler, The Middle Voice : Steady and Discrete Manifolds in Walter Benjamin, Working Paper 3.25, Center for German and European Studies, UC Berkeley, 1996. Pour tout ce paragraphe : ibid., p. 3 et suiv. Dans son célèbre essai sur le langage, Walter Benjamin parle de l'être spirituel qui se communique dans un langage particulier. Mais pour «se communiquer », il doit être à l'intérieur du procès de communication. 
Dans ce médium (qui n'a rien à voir avec les " médias » omniprésents de nos jours), « il n'y a ni sujets, ni objets, parce qu'il [le médium] est un espace de transparence [translucence] et de perméabilité. C'est un espace au sens strict du mot : à savoir non pas seulement une étendue limitée à l'intérieur d'une extension illimitée, mais un espace dépourvu de dehors ${ }^{37}$ ». Tout est fait de langage, et il n'y a rien qui puisse se situer au-dehors. Autre variante de la fameuse phrase : il n'y a pas de métalangage.

Nous pouvons peut-être entendre la différance en des termes similaires, et dire que la différance de Derrida est " un espace dépourvu de dehors ». C'est un médium, comme l'air, ou plutôt, si je puis dire, comme le serait la respirance. Penser la différance n'est possible que dans une telle voix moyenne. C'est pourquoi Derrida écrit dans Voyous ceci qui, au premier regard, peut paraître étonnant : «De cette pensée [de la déconstruction, de cette pensée de ce qu'il appelle khora] on ne peut sans doute déduire aucune politique, aucune éthique et aucun droit. Bien sûr, on ne peut rien en faire. On n'a rien à en faire. Mais irait-on jusqu'à en conclure que cette pensée ne laisse aucune trace sur ce qu'il y a à faire - par exemple dans la politique, l'éthique ou le droit à venir ${ }^{38}$ ? »

C'est dans des phrases comme celles-là que beaucoup de commentateurs voient la preuve d'une absence d'engagement politique. Mais il n'en est rien. Les conséquences de la conception du langage selon la mystique juive et selon Benjamin sont énormes : tout est issu de langage, tout participe du langage. Par conséquent, jurer, abuser du langage porte atteinte à la matière même dont le monde est fait, à toutes les créatures, y compris les choses dites inanimées. De même, les conséquences de la conception de la différance comme médium au sens de Benjamin sont énormes.

Pour le clarifier, tournons-nous vers « Avouer - 1'Impossible : "Retours", repentir et réconciliation ${ }^{39}$ ». Dans cette « leçon », Derrida poursuit une méditation sur ce que c'est que « vivre ensemble », en dis-

L'être qui est désigné par le «se » est cette intériorité. Autrement dit, pour Benjamin, le langage se communique, et on ne peut pas penser cela dans la voix active, ni dans la voix passive. Le langage qui se communique opère dans la voix moyenne, ou dans ce que Benjamin appelle le médium qui ne doit pas être confondu avec le moyen, l'outil ou l'instrument par lequel on achève, par exemple, un but (Mittel).

${ }^{37}$ W. Kittler, The Middle Voice, op. cit., p. 4.

38 Voyous, op. cit., pp. 14-15. «On ne peut rien en faire », parce qu'un tel « faire » n'est possible qu'avec une opposition de sujet et d'objet.

39 Jacques Derrida, « Avouer - l'Impossible : "Retours", repentir et réconciliation », dans Colloque des intellectuels juifs de langue française, Comment vivre ensemble? Actes du XXXVII ${ }^{e}$ Colloque des intellectuels juifs de langue française, Textes réunis par Jean Halpérin et Nelly Hansson, Paris, Albin Michel, 2001, p. 181 et suiv. 
tinguant le nom « l'ensemble » de l'adverbe « ensemble ». Permettez-moi de paraphraser ce texte et de le citer in extenso.

Le nom « l'ensemble » est clairement mis du côté de l'identité assumée, par conséquent du côté de l'être, de la souveraineté, de la maîtrise, du côté, donc, de l'exclusion de ce qui n'appartient pas à un tel ensemble identique ou identitaire. En revanche, « l'adverbe, dans l'expression "vivre ensemble", semble ne trouver son sens et sa dignité que là où il excède, disloque, conteste l'autorité du nom "ensemble", à savoir la clôture d'un ensemble, fût-ce l'ensemble d'un "vivant", d'un système, d'une totalité [...] » (p. 185). Autrement dit, selon Derrida, « l'autorité de l'ensemble sera toujours la première menace pour tout "vivre ensemble". Et inversement, tout "vivre ensemble" sera la première protestation ou contestation, le premier témoignage contre l'ensemble » (p. 185).

Un peu plus loin dans son texte, Derrida précise que « quelque éthique du "vivre ensemble" " suppose « l'accord par-delà toute condition statutaire, non pas forcément en contradiction avec elle, mais par-delà et à travers la normalité du lien juridique, politique, étatique, entre deux ou plus d'un ou d'une qui ne sont pas seulement des époux, $[\ldots]$ des compatriotes, [...] des coreligionnaires, mais restent des étrangers, des autres et radicalement autres. La paix du "vivre ensemble" excède donc le droit, voire le politique, en tout cas le politique déterminé par l'étatique, par la souveraineté de l'Etat » (p. 193).

Ce « vivre ensemble » « ouvre une autre dimension » à la «nécessité » du « il faut bien vivre ensemble » que l'on peut entendre dans deux sens très différents que Derrida explique en détail. "Le "bien" du "bien vivre ensemble" suppose l'interruption du rapport naturel autant que conventionnel; il suppose même cette interruption tout court qu'on appelle la solitude absolue, la séparation, le secret inviolable » (p. 193 et suiv.)

" [...] on ne dira pas que les parties d'un même ensemble naturel, organique et vivant vivent ensemble. L'adverbe "ensemble" dans l'expression "vivre ensemble" ne renvoie plus à la totalité d'un ensemble naturel, biologique ou génétique, à la cohésion d'un organisme ou de quelque corps social (famille, ethnie, nation), qui serait mesurée à cette métaphore organique. "Vivre ensemble" suppose donc un excès interrupteur et au regard de la convention statutaire, du droit, et au regard de la symbiose, du vivre ensemble symbiotique, grégaire ou fusionnel. [...] Voilà donc une double et paradoxale prescription. Elle est inscrite dans l'idiome, c'est-à-dire déjà dans un mode du vivre-ensemble. "Vivre ensemble" ne se réduit ni à la symbiose organique ni au contrat juridico-politique. $\mathrm{Ni}$ à la "vie" selon la nature ou la naissance, le sang ou à la terre, ni à la vie selon la convention; le contrat ou l'institution. "Vivre ensemble", si c'était possible, ce serait mettre à l'épreuve l'insuffisance de ce vieux couple de concepts qui conditionne en Occident à peu près toute métaphy- 
sique, toute interprétation du lien social, toute philosophie politique ou toute sociologie de l'être-ensemble, le vieux couple physis/nomos, physis/ thesis, nature/convention, vie biologique/droit - droit que je distingue ici, plus que jamais, de la justice et de la justice du "vivre ensemble". On ne pensera le "vivre ensemble", et le "vivre" du "vivre ensemble" et le "comment ensemble" qu'en se portant au-delà de tout ce qui se fonde sur cette opposition nature/culture. C'est-à-dire au-delà de tout, à peu près tout » (p. 194).

Y compris, bien sûr, l'opposition humain/animal et la hiérarchie qui lui est étroitement associée.

Pour explorer les modalités du « vivre ensemble », venons-en, brièvement, à un texte de Kafka, Joséphine la cantatrice ou le Peuple des souris $^{40}$. C'est un texte dont le titre implique déjà que la façon dont on pense Joséphine sera déterminante pour la façon dont on pense «le peuple des souris ». Bien entendu, je n'ai pas le temps de commenter ce texte dans le détail, mais il me semble que ce récit est une mise en scène d'un vivre ensemble dans lequel «l'ensemble » nominal est interrompu en faveur de l'adverbe " ensemble ». Pardonnez encore une fois la citation de longs extraits qui me paraissent nécessaires pour mon argument.

Le lecteur n'apprend jamais le nom ou d'autres caractéristiques de celui ou celle qui raconte, sauf qu'il ou elle appartient au peuple des souris. Il s'agit donc d'un peuple d'animaux, d'animaux parlants, et qui s'exprime de préférence en sifflant. Joséphine la cantatrice a, selon elle, perfectionné ce moyen d'expression quotidien en en faisant un art. Selon elle, son sifflement n'est pas un sifflement, mais un chant. Et elle veut être reconnue pour l'exceptionnelle beauté de son art du chant, en dépit $\mathrm{du}$ fait que, selon la narratrice, son sifflement ne se distingue guère de celui des autres souris, sauf, peut-être, qu'il est un peu plus faible. ${ }^{41}$

Néanmoins, comme la souris narratrice le dit dans sa troisième phrase, " il n'y a personne » que son chant « ne transporte, ce qui est d'autant plus remarquable que notre espèce (Geschlecht) ne témoigne en général

40 Franz Kafka, « Josefine, die Sängerin oder Das Volk der Mäuse », dans: Franz Kafka, Sämtliche Erzählungen, édité par Paul Raabe, Frankfurt: Fischer 1970/1992, p. 172 et suiv. ; "Joséphine la Cantatrice ou le Peuple des souris », dans La Colonie pénitentiaire et autres récits, traduit de l'allemand par Alexandre Vialatte, Paris, Gallimard, 1948, pp. 86--110. Les commentaires de ce texte sont nombreux. Mentionnons-en seulement deux qui ont particulièrement influencé ma lecture : Joseph Vogl, Ort der Gewalt. Kafkas literarische Ethik, München, Fink 1990, pp. 221 et suiv., et Wolf Kittler, Der Turmbau zu Babel und das Schweigen der Sirenen : über das Reden, das Schweigen, die Stimme und die Schrift in vier Texten von Franz Kafka, Erlangen, Palm \& Enke, 1985, pp. 181 et suiv.

${ }^{41}$ Edition allemande, p. 173; traduction française, p. 87. Par la suite, les deux paginations seront indiquées entre parenthèses dans le texte. 
d'aucun penchant pour la musique » $(172 / 85)$. La « puissance » du chant de Joséphine a donc un « grand effet », en dépit du fait que « la paix et le silence [littéralement, la paix silencieuse] sont notre plus chère musique » (172 et suiv./85). Pourtant, quand Joséphine annonce qu'elle va chanter, le peuple ne dit pas non : il se rassemble pour l'écouter. Il reconnaît son art, mais, nuance décisive, il s'abstient, calmement, de reconnaître l'exceptionalité de cet art. Autrement dit, le peuple refuse de dire non, refuse de faire honte à Joséphine, mais en même temps s'oppose à son désir de gouverner, son désir de royaume, son désir d'être une artiste exceptionnelle, qui pourrait par son art «sauver » le peuple.

Après une description détaillée du « sifflement » (Pfeifen) du peuple des souris, et donc, en fait, de la banalité du prétendu " chant » de Joséphine, la souris narratrice pose la question de savoir pourquoi le peuple fait tant d'efforts pour la satisfaire. Elle dit que cette question est directement liée à « la question du chant» de Joséphine. Et elle constate qu'on pourrait éliminer la première de ces questions et n'en faire qu'une avec la seconde, « si l'on pouvait affirmer que le peuple est inconditionnellement dévoué à Joséphine pour l'amour de son chant» $(176 / 92)^{42}$. Mais, continue la souris narratrice, «ce n'est justement pas le cas ». Suit une explication décisive : « Notre peuple ne connaît guère de dévouement inconditionnel (Ergebenheit) ; ce peuple, qui aime par-dessus tout la ruse (la ruse innocente, les chuchotements puérils, les petits potins - sans méchanceté ! - qui se susurrent du bout des lèvres), ce peuple ne peut, quand même, pas se donner (sich hingeben) inconditionnellement, Joséphine le sent bien elle-même; c'est ce qu'elle combat de tout l'effort de son faible gosier » (p. 176/92) ${ }^{43}$.

Et la narratrice d'enchaîner : "Évidemment, dans ces jugements généraux il ne faut pas aller trop loin : le peuple est malgré tout dévoué (ergeben : aussi, "soumis"] à Joséphine, je dis seulement qu'il ne l'est pas inconditionnellement ${ }^{44}$. Il ne serait pas capable, par exemple, de rire d'elle. On peut bien se l'avouer : il y a des choses chez Joséphine qui en donneraient envie ; et, d'une façon générale, nous sommes toujours prêts à le faire ; en dépit de notre misère, un léger rire trouve toujours sa place chez nous ; mais de Joséphine, nous ne rions pas » (176/92 et suiv.). Car « on ne rit pas de ce qui vous est confié ; ce serait contraire au devoir (Pflichtverletzung) ; la pire méchanceté que les plus méchants d'entre nous puissent se permettre à l'égard de Joséphine est de dire parfois : "Rien que de la regarder, cela nous coupe le rire". " (p. 176/93)

\footnotetext{
42 Traduction légèrement modifiée.

43 Traduction légèrement modifiée.

${ }^{44}$ La traduction de Viallatte lit « sans restriction ».
} 
C'est alors que la narratrice compare la façon dont le peuple " veille » sur Joséphine avec la relation d'un enfant avec son père - sauf que cette relation est tout sauf paternaliste. Par exemple, le rôle du père n'est pas ici de gouverner ni de commander : " On pourrait penser que notre peuple n'est pas apte à remplir des devoirs si paternels, mais en réalité, tout au moins dans ce cas, il les remplit de façon exemplaire : nul d'entre nous (kein Einzelner) ne saurait faire isolément ce que le peuple réalise là dans son ensemble. Evidemment, la différence de force (Kraftunterschied) entre le peuple et l'individu est si énorme (ungeheuer), il suffit que le peuple mette son protégé (Schützling) dans la chaleur de sa proximité, et il est suffisamment protégé (beschützt genug) $(176 / 93)^{45}$. »

Le peuple, tranquillement et sans amertume ni ressentiment, refuse ou réfute le double désir de commander et d'être commandé, en re-définissant la figure du père d'une façon qui est, dans la tradition occidentale, extraordinaire : c'est le collectif, l'ensemble, qui protège ensemble le Schützling, le protégé.

Du début à la fin, "l'ensemble » de ce peuple est décrit non pas comme une identité monolithique, mais, au contraire, constamment en mouvement, dans une dissémination incessante, reflétée par les bruits émis par son langage : un langage fait de chuchotements, de sifflements, de bavardages et de rires. Un peuple qui possède un tel langage ne peut pas se soumettre, se rendre ni se dévouer inconditionnellement.

Joséphine est convaincue d'être la salvatrice de son peuple. A quoi la narratrice répond : "Il est facile, dis-je, de se poser après coup en sauveur de ce peuple qui s'est toujours sauvé lui-même, plus ou moins, fût-ce au prix de sacrifices devant lesquels les historiens - nous négligeons en général la science de l'histoire complètement - restent pétrifiés d'épouvante. Et cependant il n'en est pas moins que c'est précisément dans nos périodes de détresse que nous prêtons le mieux l'oreille à la voix de Joséphine » (177/94 et suiv. $)^{46}$.

Et pourquoi ? La narratrice précise : «C'est comme si [je souligne comme si] nous buvions encore rapidement - oui, la hâte est nécessaire, Joséphine l'oublie trop souvent - ensemble (gemeinsam) une coupe de paix avant le combat. Il s'agit moins, alors, d'un concert que d'une réunion du peuple et d'une réunion où le silence est complet, à l'exception du petit sifflement au-devant; l'heure est beaucoup trop grave pour qu'on la gaspille en bavardages » $(177 / 95)^{47}$.

\footnotetext{
45 Traduction légèrement modifiée.

46 Traduction légèrement modifiée.

47 Traduction modifiée.
} 
Une page plus loin, la narratrice ajoute une phrase extraordinaire : le chant de Joséphine, ou plutôt son sifflement, dit-elle, " arrive presque à chacun comme un message du peuple ; le grêle sifflet de Joséphine, au milieu des décisions difficiles, est presque comme la pauvre existence de notre peuple dans le tumulte d'un monde ennemi. Joséphine s'affirme (behauptet sich), ce rien de voix, ce rien de performance, s'affirme et s'ouvre un chemin jusqu'à nous, il fait du bien d'y penser ${ }^{48} »(178 / 96)$.

Voilà comment cette souris singulière est reconnue : son chant n'est pas un salut, ni l'exception qu'il faut adorer, mais le message du peuple à tout un chacun, ou, plutôt, non pas un message identique, identificateur, mais sa promesse messianique sans messie : presque comme un message.

Et la narratrice continue par une déclaration qui pourrait presque couper le souffle : «Si jamais il devait se trouver parmi nous un véritable artiste du chant (Gesangskünstler), nous ne le supporterions certainement pas dans ces moments-là »- à savoir en temps de nécessité (et ce peuple ne vit qu'en des temps pareils) - « et nous rejetterions d'une voix unanime l'absurdité d'une telle performance » $(178 / 96)^{49}$. Kafka juxtapose ici l'absurdité, la Unsinnigkeit, littéralement l'absence de sens (Vialatte traduit par « l'insanité ») à l'unanimité : « einmütig », littéralement, l'union des cœurs. Avoir un véritable artiste, quelqu'un qui fait des choses exceptionnelles, qui assume un rôle d'exception, de souveraineté, peut-être donc qui jouit de la gloire de commander, n'a littéralement aucun sens pour ce peuple. Souvenez-vous du lieutenant Cabrita à l'heure de sa mort et juste avant son délire où il revit la bataille d'Alcacer-Quibir, qui fait entendre, de façon si terrifiante, le NON : "Vérité ! Secrète et inexplicable. Au lieu d'un sens logique, cette vérité a un sens dernier qui explique tout ${ }^{50}$. » Le un sens et le tout expliqué, le « un » et l' " universel », le « un » et l'ensemble nominal vont donc, précisément, ensemble, inextricablement.

À l'opposé, ce qui a du sens pour le peuple des souris, c'est une sorte d'union des cœurs qui ne peut que rejeter un "sauveur » et son autorité qui commandent. Le texte ne laisse aucun doute sur le fait que c'est cette position exceptionnelle de celui qui commande à laquelle Joséphine aspire.

Le peuple rejette cette position, ensemble. Non pas comme un ensemble qui commande, mais depuis et dans un vivre ensemble. Car la raison de ce refus est précisément située dans "unserer Lebensweise 》, notre façon de vivre (178/97), autrement dit, notre façon de vivre ensemble.

Il est très significatif que partout dans le texte de Kafka la façon de

\footnotetext{
48 Traduction légèrement modifiée. Je souligne (E.W).

49 Traduction légèrement modifiée.

${ }^{50}$ Je souligne, E.W.
} 
vivre soit inséparable des façons de parler et de penser. C'est, si je puis dire, une seule et même façon d'être, parce que la façon de parler est une production non pas d'un sens unique, mais de bruits, de chuchotements et de rires, qui fait écho aux multiples mouvements du peuple des souris. En bref, cette " façon » est un médium dont, dans le peuple des souris, il n'y a pas de dehors, même pas pour la cantatrice Joséphine. Et ce médium donne «lieu », si je puis dire, à la différance. Ce n'est pas la production d'un sens unique, mais la façon de vivre ensemble qui, en fait, réfute le désir de royaume.

Cette façon de vivre est décrite comme " dispersée (ou disséminée)» par « nécessité économique » (p. 179/97), et, à l'occasion, sinnlos (dépourvu de sens, absurde, mais je l'entends dans l'acception de sans but ultime gouvernant toutes les actions), verschwenderisch (gaspillant, au sens d'un don sans compter), grosszügig (magnanime), leichtsinnig (intrépide, risqué, au sens de "sans réfléchir»; littéralement, avec un « sens léger ») (p. 179/98 $\left.{ }^{51}\right)$. Pensons à l'ami décrit par Nietzsche comme celui qui donne d'un cœur « sur-plein » ou débordant (et c'est le " sur» de ce « sur-plein » qui définit le « sur »-homme) $)^{52}$.

Le langage d'un tel peuple, qui chuchote, rit, bavarde et se meut continuellement, pourrait ressembler au yiddish, que Kafka décrit comme un langage en perpétuel mouvement, qui refuse de se laisser apprivoiser par les grammairiens. Ce langage ne consiste qu'en "mots étrangers », est toujours parlé, sans répit ${ }^{53}$. (Quel cauchemar, pourrions-nous ajouter, pour les gardiens de la langue d'une institution comme l'Académie française !)

Un tel langage ne produit pas, pour revenir à « La différance », de « mot unique », de « nom unique », de " maître-nom » ou de " nom de l'être ». Et, comme l'écrit Derrida, « il faut le penser sans nostalgie, c'est-à-dire hors du mythe de la langue purement maternelle ou purement paternelle, de la patrie perdue de la pensée. Il faut au contraire l'affirmer, au sens où Nietzsche met l'affirmation en jeu, dans un certain rire et dans un certain pas de la danse ${ }^{54} »$.

Le langage du peuple des souris produit du sens non pas à cause de maîtres-noms, mais dans l'événement de son énonciation.

${ }^{51}$ La traduction française s'éloigne ici considérablement du texte de Kafka : « [...] nous agissons parfois follement; et de la façon précisément dont les enfants agissent follement: comme de petits sots, de petits gâcheurs, de petits prétentieux: et cela pour une babiole » (p. 98).

52 Friedrich Nietzsche, Also sprach Zarathustra, Kritische Studienausgabe, vol. 4, éd. Giorgio Colli et Mazzino Montinari, München, Deutscher Taschenbuch Verlag 1988, p. 78.

${ }^{53}$ Franz Kafka, « Rede über die jiddische Sprache », dans: Franz Kafka, Hochzeitsvorbereitungen auf dem Lande und andere Prosa aus dem Nachlass, Frankfurt, Fischer, 1983, p. 306.

54 « La diférance », art. cité, p. 28 et suiv. 
Permettez-moi d'ajouter que si le peuple des souris interrompt et rejette sans ressentiment les aspirations à l'exception de la cantatrice Joséphine, Joséphine la cantatrice, à son tour, empêche ce qui pourrait devenir une réunion solennelle du peuple : le moindre bruit, la moindre panne, la moindre interférence sont considérés par elle aptes « à rehausser l'effet de son chant : ne chante-t-elle pas à son avis devant des sourds ? On ne lui refuse ni l'enthousiasme ni les applaudissements : mais il y a longtemps, pense-t-elle, qu'elle n'a plus à attendre de nous une véritable compréhension. Aussi tous les incidents sont-ils bienvenus; tout ce qui s'oppose du dehors à la pureté de son chant, et est vaincu par un combat facile, voire sans combat, par simple contraste, peut contribuer à éveiller la foule, à lui enseigner sinon la compréhension, au moins le respect intuitif (ahnungsvollen Respekt)» (174/89 et suiv. ${ }^{55}$ ).

Donc, même pour Joséphine, il ne s'agit pas d'une production de « compréhension » (Verständnis). L'ensemble du « vivre ensemble » d'un tel « peuple » pourrait être décrit dans les termes d'une « discorde "active", en mouvement, des forces différentes et des différences de forces ».

C'est dans ces termes-là que Derrida énonce sa cinquième définition de « la différance » dans le texte de 1968 : « Nous pourrons donc appeler différance cette discorde "active", en mouvement, des forces différentes et des différences de forces que Nietzsche oppose à tout le système de la grammaire métaphysique partout où elle commande la culture, la philosophie et la science ${ }^{56}$. »

Si la différance peut être décrite comme le médium depuis lequel les oppositions, les différences déterminées deviennent possibles, ensemble comme adverbe, comme dans vivre ensemble, peut être décrit comme le médium depuis lequel tout ensemble ne peut que se constituer ; autrement dit, depuis cet autre " ensemble » adverbial qui toujours travaillera l'ensemble nominal depuis son «propre » intérieur, le fissurant pour l'ouvrir vers à l'adverbe.

Et puisque Derrida se réfère ici à Nietzsche, arrêtons-nous, trop brièvement, sur ce court texte de Ainsi parlait Zarathoustra, sur l'amour du prochain : «Est-ce que je vous conseille l'amour du prochain (Nächstenliebe)? Je vous conseille plutôt de fuir le prochain et d'aimer le plus lointain, l'amour du plus loin (Fernstenliebe).

«Plus haut que l'amour pour le prochain (Liebe zum Nächsten) est l'amour pour le plus loin et pour celui-à-venir (zum Künftigen) ; plus haut que l'amour pour les humains (Menschen) vaut pour moi l'amour pour des choses et des spectres. [...] Je ne vous enseigne pas le prochain,

55 Traduction modifiée.

56 J. Derrida, « La différance », art. cité, p. 19. 
mais l'ami. Que l'ami vous soit la fête de la terre et le pressentiment du sur-homme (Vorgefühl des Übermenschen). Je vous enseigne l'ami et son cœur sur-plein (und sein übervolles Herz) ${ }^{57}$. "

Si différents qu'ils paraissent au premier regard, l'ami de Nietzsche et le peuple des souris de Kafka ont des traits communs : ils donnent sans compter (verschwenderisch), d'un cœur " sur-plein », et ils vivent dans l'affirmation. En tenant compte d'autres passages d'Ainsi parlait Zarathoustra, nous pouvons en déduire que l'ami nietzschéen aime le rire pas moins que le peuple des souris.

Mais pourquoi Zarathoustra enseigne-t-il l'amour des choses et des spectres ? Peut-être parce qu'un tel amour défie les oppositions métaphysiques telles que Derrida les énumère dans le passage déjà cité de "Avouer... ». Autrement dit, l'amour des choses et des spectres s'approche bien plus du vivre ensemble décrit par Derrida, parce que les ensembles s'y trouvent brisés, parce que la " naturalité ", « l'homogénéité » ou la proximité alléguées de « l'amour » ou de " l'amitié » s'y trouvent suspendues ${ }^{58}$.

Nietzsche n'a aucun doute sur la violence de l'ensemble des prochains : " Les plus lointains doivent payer votre amour du prochain, et déjà quand cinq de vous sont ensemble, un sixième doit toujours mourir ${ }^{59}$. " L'ensemble des prochains est un ensemble de ceux qui se connaissent, ou croient se connaître, un ensemble formé par la réciprocité ${ }^{60}$. C'est, selon Nietzsche, un ensemble dont le prix est la mort du plus lointain, de celui qui se trouve en dehors de l'ensemble des " prochains ». Quant aux choses, elles n'aiment pas « en retour », ni dans la réciprocité. L'amour pour elles est un amour en dehors de toute économie de la réciprocité. En outre, l'amour des choses comme l'amour des spectres défient tout ensemble qui se définirait exclusivement par « le vivant».

Lisons Derrida : "Vivre ensemble" avec des morts, [...] c'est une possibilité essentielle de l'existence. Elle nous rappelle que dans "vivre ensemble" l'idée de vie n'est ni simple ni dominante bien qu'elle reste irréductible. "Vivre ensemble" avec le passé de ceux qui ne sont plus et ne seront plus présents ou vivants, ou avec l'avenir imprévisible de ceux qui ne sont pas encore vivants au présent, si cela constitute une possibilité tout aussi irrécusable de l'être-avec-soi, d'un "vivre ensemble" - avec-soi, en un soi ainsi partagé ou divisé, enclavé, multiplié ou déchiré, ouvert

${ }^{57}$ Friedrich Nietzsche, Also sprach Zarathustra, op. cit., p. 77 et suiv.

58 J. Derrida, Politiques de l'amitié, op. cit., p. 128. Cf. aussi chapitres 2 et 3, qui portent sur l'amitié chez Nietzsche.

${ }^{59}$ Friedrich Nietzsche, Also sprach Zarathustra, op. cit., p. 78.

${ }^{60}$ On se rappelle le rejet par Emmanuel Levinas de la pensée de la réciprocité de Martin Buber. 
aussi, en tout cas anachronique dans son présent même, augmenté à la fois et disloqué par le deuil ou la promesse de l'autre en soi, d'un autre plus grand, plus vieux ou plus jeune que soi, d'un autre hors de soi en soi, alors "vivre ensemble" n'a plus la simplicité du "vivre" au présent pur et simple, pas plus que la cohésion, la coïncidence avec soi d'un ensemble présent, présent vivant, présent à soi, en synchronie avec soi, conjoint à lui-même dans une sorte de totalité. L'altérité d'un passé et d'un futur irréductibles soustrait le "vivre ensemble" à la plénitude d'une présence à soi ou d'une identité » (p. 184 et suiv.).

Pour finir, permettez-moi de revenir à une assertion avancée plus haut : les conséquences de la conception de la différance comme médium sont énormes.

$\mathrm{Si}$, en effet, la différance est un médium sans dehors, nous pouvons dire que pour notre vivre-ensemble, " il ne saurait y avoir de "comment" qui précède, comme le ferait un savoir, la décision ou la responsabilité dont chacun, singulièrement, élu sans élection, élu à une place irremplaçable, doit inventer la règle $\left.{ }^{61} /\right\rangle$.

« Élu sans élection » : cela aussi, il « faut le penser sans nostalgie ${ }^{62}$ », peut-être comme Joséphine, dont la narratrice dans le texte de Kafka dit tout à la fin qu'elle sera "peut-être [...] délivrée " des peines réservées, à son avis, aux "élus ", et qu'elle " ira se perdre joyeusement dans l'innombrable foule des héros de notre peuple » ${ }^{63}$, peut-être. "Élu sans élection » : la délivrance du désir de royaume nous mène, dans le texte de Kafka, vers un hérö̈sme disséminé, innombrable. Peut-être cet héroïsme se caractérise-t-il par une responsabilité selon laquelle « chacun, singulièrement » est " élu à une place irremplaçable ", chacun, chacune d'entre nous.

Mes chaleureux remerciements vont à Madame le Professeur Fernanda Bernardo et ses collègues et étudiants pour m'avoir accueillie dans leur séminaire. Je tiens à dire ici ma profonde gratitude envers Isabelle Châtelet pour son aide précieuse dans la mise au point du manuscrit pour la publication.

61 Jacques Derrida, « Avouer - 1'Impossible : "Retours”, repentir et réconciliation », art. cité, p. 213.

62 « La différance », art. cité, p. 29.

${ }^{63}$ Franz Kafka, «Joséphine la Cantatrice ou le Peuple des souris », op. cit., p. 109 et suiv. ; traduction modifiée. 